\title{
Comparative genomics of koala, cattle and sheep strains of Chlamydia pecorum
}

\author{
Nathan L Bachmann ${ }^{1}$, Tamieka A Fraser ${ }^{2}$, Claire Bertelli, ${ }^{2,3,8}$, Martina Jelocnik ${ }^{2}$, Amber Gillett ${ }^{4}$, Oliver Funnell ${ }^{5}$, \\ Cheyne Flanagan ${ }^{6}$, Garry S A Myers ${ }^{7}$, Peter Timms ${ }^{1,2}$ and Adam Polkinghorne ${ }^{1,2^{*}}$
}

\begin{abstract}
Background: Chlamydia pecorum is an important pathogen of domesticated livestock including sheep, cattle and pigs. This pathogen is also a key factor in the decline of the koala in Australia. We sequenced the genomes of three koala C. pecorum strains, isolated from the urogenital tracts and conjunctiva of diseased koalas. The genome of the C. pecorum VR629 (IPA) strain, isolated from a sheep with polyarthritis, was also sequenced.

Results: Comparisons of the draft C. pecorum genomes against the complete genomes of livestock $C$. pecorum isolates revealed that these strains have a conserved gene content and order, sharing a nucleotide sequence similarity $>98 \%$. Single nucleotide polymorphisms (SNPs) appear to be key factors in understanding the adaptive process. Two regions of the chromosome were found to be accumulating a large number of SNPs within the koala strains. These regions include the Chlamydia plasticity zone, which contains two cytotoxin genes (toxA and toxB), and a $77 \mathrm{kbp}$ region that codes for putative type III effector proteins. In one koala strain (MC/MarsBar), the toxB gene was truncated by a premature stop codon but is full-length in IPTaLE and DBDeUG. Another five pseudogenes were also identified, two unique to the urogenital strains C. pecorum MC/MarsBar and C. pecorum DBDeUG, respectively, while three were unique to the koala C. pecorum conjunctival isolate IPTaLE. An examination of the distribution of these pseudogenes in C. pecorum strains from a variety of koala populations, alongside a number of sheep and cattle C. pecorum positive samples from Australian livestock, confirmed the presence of four predicted pseudogenes in koala C. pecorum clinical samples. Consistent with our genomics analyses, none of these pseudogenes were observed in the livestock C. pecorum samples examined. Interestingly, three SNPs resulting in pseudogenes identified in the IPTaLE isolate were not found in any other C. pecorum strain analysed, raising questions over the origin of these point mutations.
\end{abstract}

Conclusions: The genomic data revealed that variation between C. pecorum strains were mainly due to the accumulation of SNPS, some of which cause gene inactivation. The identification of these genetic differences will provide the basis for further studies to understand the biology and evolution of this important animal pathogen.

Keywords: Chlamydia pecorum, Single nucleotide polymorphism, Pseudogene, Cytotoxin

\section{Background}

Chlamydia are widely distributed and highly successful bacterial pathogens that only replicate inside eukaryotic cells, which is a key factor in their ability to remain hidden from the host immune response and to cause persistent infections [1]. Seemingly contrary to this highly

\footnotetext{
* Correspondence: apolking@usc.edu.au

${ }^{1}$ Faculty of Science, Health, Education and Engineering, University of the Sunshine Coast, Sippy Downs 4558, Queensland, Australia

${ }^{2}$ Institute for Health and Biomedical Innovation, Queensland University of Technology, Kelvin Grove 4059, Queensland, Australia

Full list of author information is available at the end of the article
}

adapted intracellular lifestyle, the majority of the eleven currently described species in the genus Chlamydia, can infect multiple host species. The best example of this is Chlamydia psittaci, primarily recognised as an avian pathogen, but is also known to infect and cause disease in cattle, sheep, pigs and horses, while posing a zoonotic risk for humans [2]. Other species such as C. pecorum, C. abortus and C. pneumoniae, can infect multiple hosts as well [3-5]. For each of these species, there is little understanding of the mechanisms involved in the adaptation to different niches, especially considering that 
different strains within a species share nearly identical genomes with a DNA sequence similarity of $>98 \%[6,7]$.

The adaptation of bacterial pathogens to specific niches is driven by the evolutionary "arms race" that takes place between the host and the bacterium [8]. The host's immune system can provide a selective pressure for the accumulation of mutations in the genes of the bacterium. Although the majority of polymorphisms within genes cause synonymous changes, which are indicative of purifying selection for the encoded protein to maintain its current function and structure, a small number of genes will accumulate non-synonymous substitutions that result in protein variation [9]. Recent data analysing the accumulation of these single nucleotide polymorphisms (SNPs) in C. trachomatis has emphasized that this will be key to understanding the host adaptation of each the chlamydial species [10].

An interesting example of chlamydial pathogenicity and intra-species host adaptation potentially lies in an analysis of the animal pathogen, $C$. pecorum. C. pecorum is a widespread pathogen of economically important livestock species such as cattle, sheep, goats and pigs. In livestock, infections of $C$. pecorum manifest as a range of diseases such as polyarthritis, pneumonia, conjunctivitis and encephalomyelitis, while also being linked to diseases of the gastrointestinal and urogenital tracts [11,12]. Beyond these reports, perhaps the most common outcome of $C$. pecorum infection is the absence of disease symptoms [13]. However, even in animals that are asymptomatic, there is evidence for a subclinical pathological effect [14]. While C. pecorum infections in livestock are of economic concern to primary producers globally, the best example of the pathogenic potential of this obligate intracellular bacterium is through the ongoing association between $C$. pecorum infection of the koala, a native Australian marsupial, and debilitating ocular and urogenital tract diseases $[15,16]$. In this capacity, C. pecorum is a key threatening process to the long-term survival of this native species [17].

In the absence of genome sequence data for the representative strains infecting each host species, efforts to understand the genetic relationship and host adaptation of $C$. pecorum strains infecting livestock and koalas has centered on the use of molecular typing methods. A previous study that investigated the molecular epidemiology of C. pecorum using a Multi Locus Sequence Analysis (MLSA) typing scheme on both livestock and koala C. pecorum strains suggested that there is limited genetic diversity between strains and that they share a common ancestor [18]. This finding was in contrast to previous reports utilising an alternative molecular target, ompA, encoding the Chlamydia major outer membrane protein, a porin responsible for nutrient transfers, attachment and structural support [19]. However, the ompA gene is likely to be evolving at a faster rate than the rest of the genome since it is located on the cell surface and, as a result, the gene is under high positive selection [20]. More recently, three $C$. pecorum strains from ruminant animals were sequenced revealing a high level of sequence similarity and gene content [21]. In this setting, it is vital to employ additional whole genome sequencing of non-ruminant C. pecorum strains in order to fully understand the genetic diversity between $C$. pecorum strains and to identify genes that could be involved in adaptation to different hosts.

In this study, the genome sequences of C. pecorum strains isolated from three koalas and a sheep were compared to identify genes that could play a potential role in adaptation to different hosts and to gain insight into genetic diversity and evolution. In addition, the koala C. pecorum genomes were compared against each other in order to examine the genetic diversity between conjunctival and urogenital $C$. pecorum strains. The survey of the broader diversity of selected regions of the C. pecorum genome in a range of clinical samples from Australian livestock and koalas was also conducted.

\section{Results}

\section{Phylogenetic relationship of $C$. pecorum as revealed by whole genome sequencing}

The draft genomes of three C. pecorum strains isolated from koalas with clinical symptoms (C. pecorum IPTaLE, C. pecorum DBDeUG and C. pecorum MC/MarsBar) each comprise a single scaffold of approximately 1.1 Mbp (Table 1). The chromosomes have a GC content of $41 \%$ and an average read coverage of $1500 \times$. The fourth draft genome was from the C. pecorum VR629 (IPA) strain that was isolated from the joint fluid of a sheep suffering from polyarthritis in the USA. None of the strains sequenced possessed the cryptic chlamydial plasmid. The three koala $C$. pecorum strains have been typed based on the ompA gene and each strain represents a different ompA serotype (Table 1) [22].

To determine the relationship between the four sequenced $C$. pecorum strains, a phylogenetic tree was constructed based on the sequence of 152 conserved genes across eight Chlamydia species. The inferred topology of the phylogenetic tree (Figure 1) is consistent with other phylogenetic analysis of Chlamydia species [23]. The phylogenetic reconstruction indicates that the closest relative of $C$. pecorum is $C$. pneumoniae. The phylogenetic analysis revealed that the three koala C. pecorum strains are part of a separate lineage from the ruminant $C$. pecorum strains sequenced in this study and those previously described $[21,24]$. The two urogenital C. pecorum strains (MC/MarsBar and DBDeUG) grouped more closely together, away from the conjunctival C. pecorum IPTaLE isolate. 
Table $1 \mathrm{C}$. pecorum draft genomes sequenced in this study

\begin{tabular}{lllll}
\hline & C. pecorum IPTaLE & C. pecorum DBDeUG & C. pecorum MC/MarsBar & C. pecorum VR629 \\
\hline Genotype & A & F & G & n/a \\
Source & Koala & Koala & Koala & Sheep \\
Year of Isolation & 2010 & 2010 & 2009 & 1968 \\
Country & Australia & Australia & Australia & USA \\
Isolation tissue & Eye & UGT & UGT & Joint \\
Disease & Conjunctivitis & UTI & Chronic cystitis & Polyarthritis \\
Total number of reads & 17011050 & 13889239 & 15011176 & 16587782 \\
Number of contigs & 14 & 8 & 14 & 5 \\
N50 & 277726 & 587274 & 575200 & 478577 \\
Assembly length (bp) & $1,090,201$ & $1,092,392$ & $1,090,698$ & $1,104,572$ \\
Number of CDS & 990 & 985 & 980 & 971 \\
\% GC content & 41 & 41 & 41 & 41
\end{tabular}

Whole genome comparison revealed a high level of gene conservation between $C$. pecorum strains from koalas and livestock

Whole genome comparisons confirmed that the four draft $C$. pecorum genomes included in this study are highly conserved and syntenic, with similar gene content. Comparisons against the genomes of other Chlamydia species revealed that C. pecorum contains several regions that differ significantly to other Chlamydia species (see Additional file 1). The variable regions include the chlamydial plasticity zone (PZ), a genomic island located near the terminus of replication and a cluster of genes that encode polymorphic membrane proteins (PMPs). Like the other $C$. pecorum genomes studied to date [21], the four draft $C$. pecorum genomes sequenced encode a near intact tryptophan biosynthesis operon but they are missing the $\operatorname{trp} E / G$ genes. The genomes of the C. pecorum strains are highly conserved with a DNA sequence identity ranging from 98.5 to $98.8 \%$. The conservation of gene content and sequence similarity between

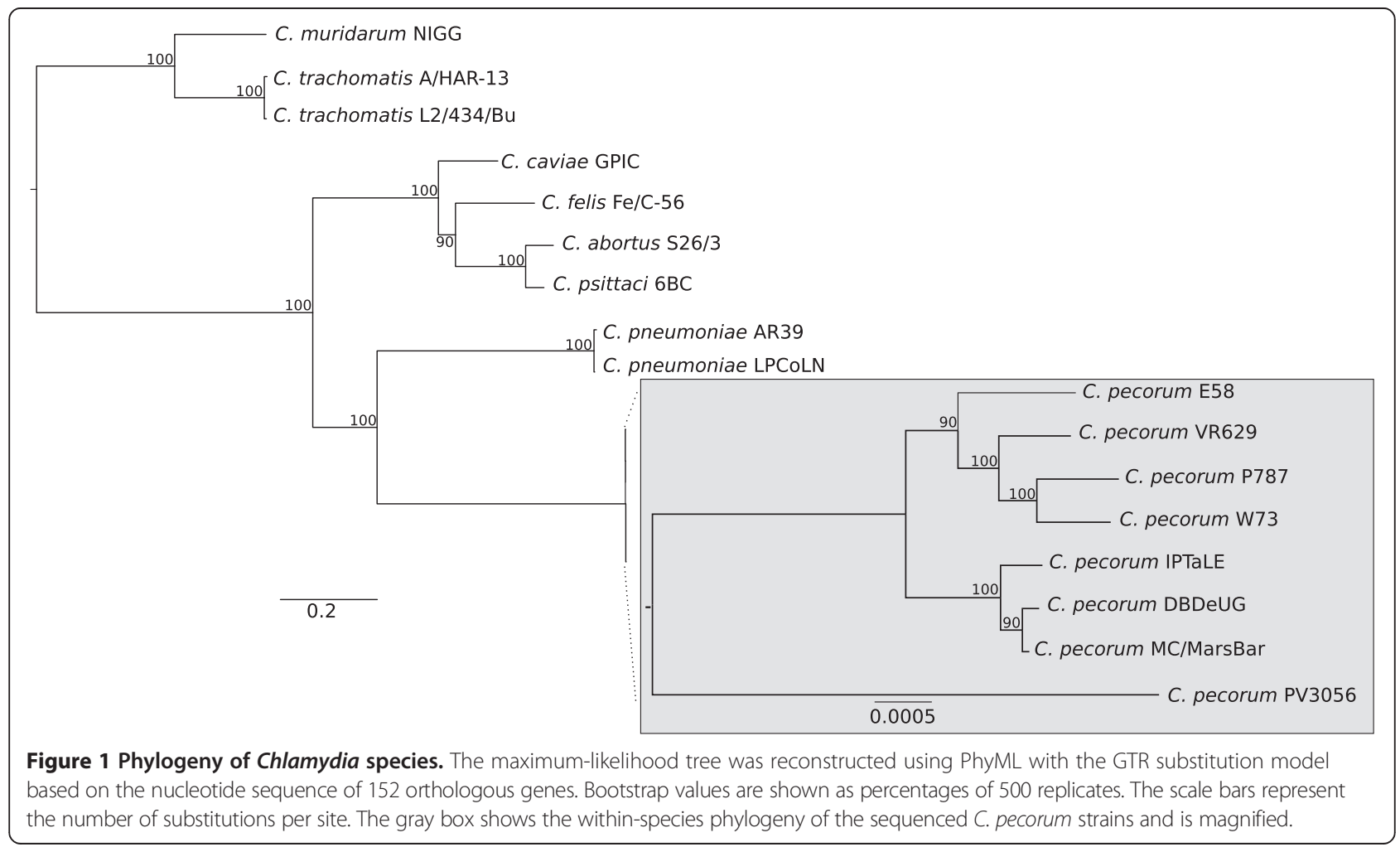


C. pecorum strains is consistent with other Chlamydia species [6,7].

\section{Single nucleotide polymorphisms contribute to the} genetic diversity between the $C$. pecorum genomes Although the overall gene content is conserved between each $C$. pecorum genome, there are a significant number of SNPs that contribute to variation between the C. pecorum strains. The number of predicted SNPs between livestock and koala $C$. pecorum strains ranges from 4914 to 6438 SNPs. Between the livestock C. pecorum strains the number of polymorphisms range from 3533 to 4129 SNPs with the exception of the phylogenetically distinct $C$. pecorum PV3056 strain which differs from the other livestock strains by 15077 SNPs. Comparisons of the three koala $C$. pecorum genomes revealed that there are 1461-1747 SNPs (Table 2). The distribution of SNPs was plotted across the whole genome using a custom $\mathrm{R}$ script to reveal regions that are accumulating mutations (Figure 2). The region with the largest number of SNPs in the $C$. pecorum genomes is a $36 \mathrm{~kb}$ gene cluster encoding 11 PMPs. Between C. pecorum E58 and C. pecorum VR629, there are 271 synonymous and 220 non-synonymous SNPs within the PMP cluster, with the majority of SNPs found in the pmpG subfamily (Figure 3). Between the livestock C. pecorum E58 and koala C. pecorum MC/MarsBar isolates, this pmp cluster contains 461 synonymous and 433 non-synonymous SNPs making this gene cluster the most variable region between $C$. pecorum strains from different hosts. Comparison between the genomes of koala C. pecorum strains indicates that most of the variation observed in this region occurs with the $p m p G$ genes. The adaptation to different hosts is likely the result of small and subtle changes that occurred in the genomes rather than differences in the gene content.

SNPs within koala $C$. pecorum strains highlight genes potentially associated with host adaptation to marsupials The distribution of SNPs across the C. pecorum genomes revealed that there are two regions in the koala and

Table 2 Total SNPs in C. pecorum genomes using C. pecorum MC/MarsBar as a reference

\begin{tabular}{lllll}
\hline Strain & Host & $\begin{array}{l}\text { No. of SNPs } \\
\text { within genes }\end{array}$ & $\begin{array}{l}\text { No. of SNPs } \\
\text { in intergenic } \\
\text { regions }\end{array}$ & $\begin{array}{l}\text { Total no. } \\
\text { of SNPs }\end{array}$ \\
\hline C. pecorum E58 & Cattle & 5454 & 483 & 5937 \\
C. pecorum PV3056 & Cattle & 5869 & 569 & 6438 \\
C. pecorum W73 & Sheep & 4526 & 430 & 4956 \\
C. pecorum P787 & Sheep & 4534 & 380 & 4914 \\
C. pecorum VR629 & Sheep & 4704 & 302 & 5006 \\
C. pecorum IPTaLE & Koala & 1619 & 128 & 1747 \\
C. pecorum DBDeUG & Koala & 1357 & 104 & 1461 \\
\hline
\end{tabular}

European livestock $C$. pecorum strains that are hotspots for the accumulation of SNPs (Figure 2). The corresponding highly polymorphic regions do not show a similar level of diversity between the sheep and cattle C. pecorum strains isolated from USA. The first region, located near the origin of replication, is approximately $77 \mathrm{kbp}$ in length. A detailed analysis of this region revealed the presence of genes that encode for putative type III effector proteins, chaperones and other potential virulence-related proteins. Also found here is a cluster of genes that encode the inner membrane components of the Type III secretion system (T3SS). In contrast to many other bacterial pathogens where the genes for the T3SS apparatus are clustered together on pathogenicity islands, Chlamydia T3SS genes are located in four separate clusters disseminated throughout the genome [26,27]. In addition, there is a sulfur transfer system encoded by the sufBCD operon and sufS similar to a system in E. coli; the rest of the genes located within this region are predicted to be involved in various metabolic processes [28].

The $77 \mathrm{kbp}$ region is a hotspot for SNPs in both the Australian koala strains (C. pecorum MC/Marsbar, C. pecorum IPTaLE and C. pecorum DBDeUG) and European livestock strains (C. pecorum PV3056, C. pecorum W37 and C. pecorum P787) but this region is conserved between the two USA livestock strains (C. pecorum VR629 and C. pecorum E58) (Figure 4). Between C. pecorum E58 and $C$. pecorum MC/MarsBar, we observed 307 synonymous and 208 non-synonymous SNPs located within genes in the $77 \mathrm{kbp}$ region. The majority of the nonsynonymous SNPs are associated with putative virulence factors, including the Type III effector proteins. A selection analysis was carried out on each gene within this region between $C$. pecorum E58 and C. pecorum $\mathrm{MC} /$ MarsBar to determine if any genes are under positive selection [29]. Between the livestock and koala strains, most of the genes in this region are under purifying selection with a $d_{n} / d_{s}$ ratio of less than 1 . However, we observed three genes that are under positive selection, including one of the putative Type III effectors (locus tags: CpecG_0280, CpecA_0283, CpecF_0282) and two conserved hypothetical proteins (see Additional file 2). Within the koala $C$. pecorum strains themselves, all the genes in this region are under purifying selection with mostly synonymous SNP differences, with the exception of the srpA1 gene (locus tags: CpecG_0278, CpecA_0281, CpeF_0280). The SrpA1 protein has $100 \%$ sequence identity between $C$. pecorum MC/MarsBar and C. pecorum IPTaLE, however it only shares $85 \%$ sequence identity to the SrpA1 homolog from C. pecorum DBDeUG (see Additional file 3). The C-terminal domain of SrpA1 was conserved across all the $C$. pecorum genomes and it is the $\mathrm{N}$-terminal domain that is variable even between C. pecorum koala strains. 


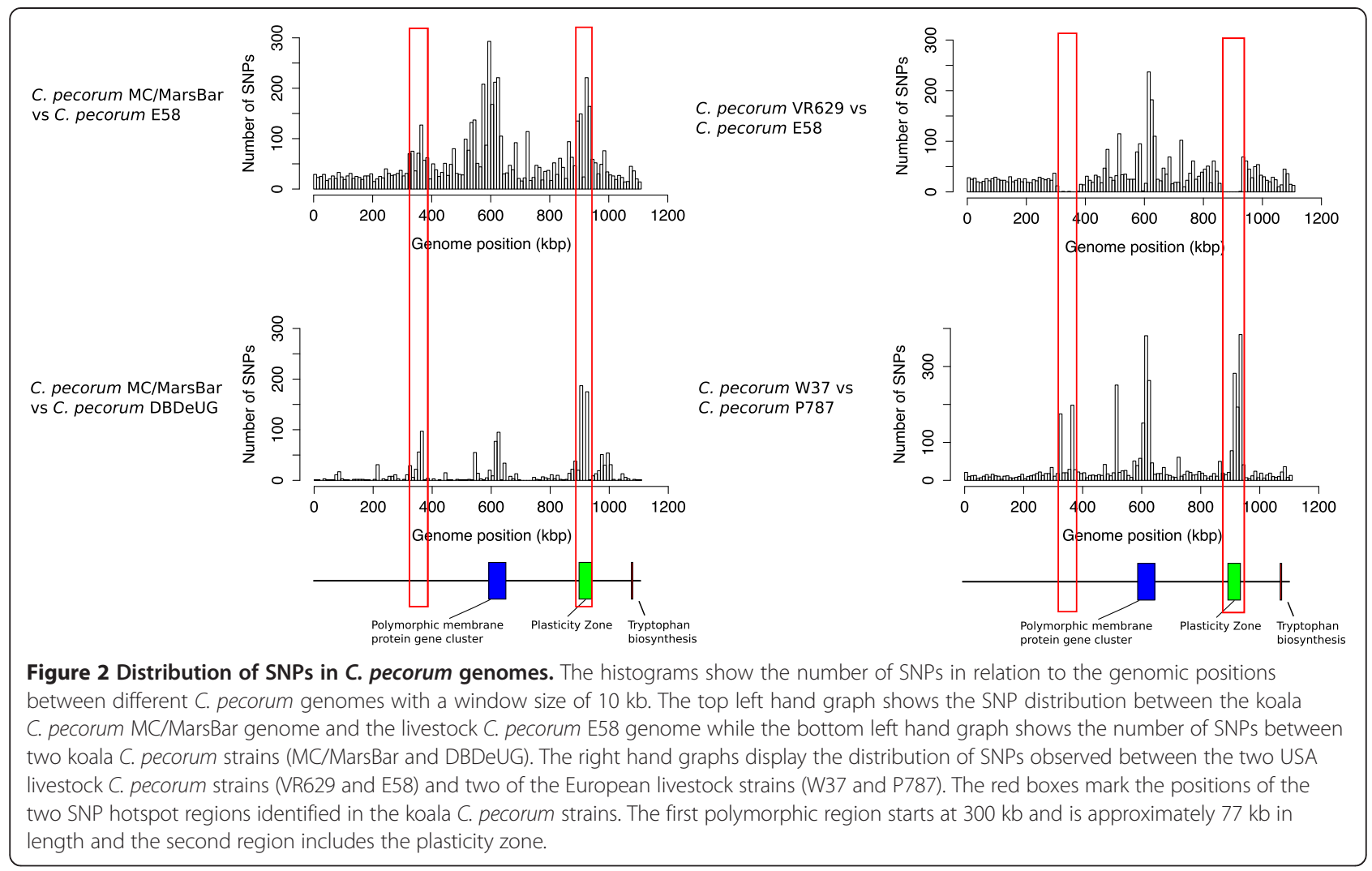

The second genomic region with a high frequency of SNPs is the PZ (Figure 5). However, it should be noted that in the koala C. pecorum genomes, there are assembly gaps within the PZ and that the number of predicted SNPs in the PZ is likely an under estimation. Paired-end data is used to estimate the size of the gaps, which suggest that the gene content of the PZ is identical in each of the genomes. In C. pecorum, the $\mathrm{PZ}$ is $42 \mathrm{~kb}$ with the acetyl-CoA-carboxylases genes $(a c c \mathrm{~B}$ and $a c c \mathrm{C})$ located at the $5^{\prime}$ boundary and the guaAB and add genes at the

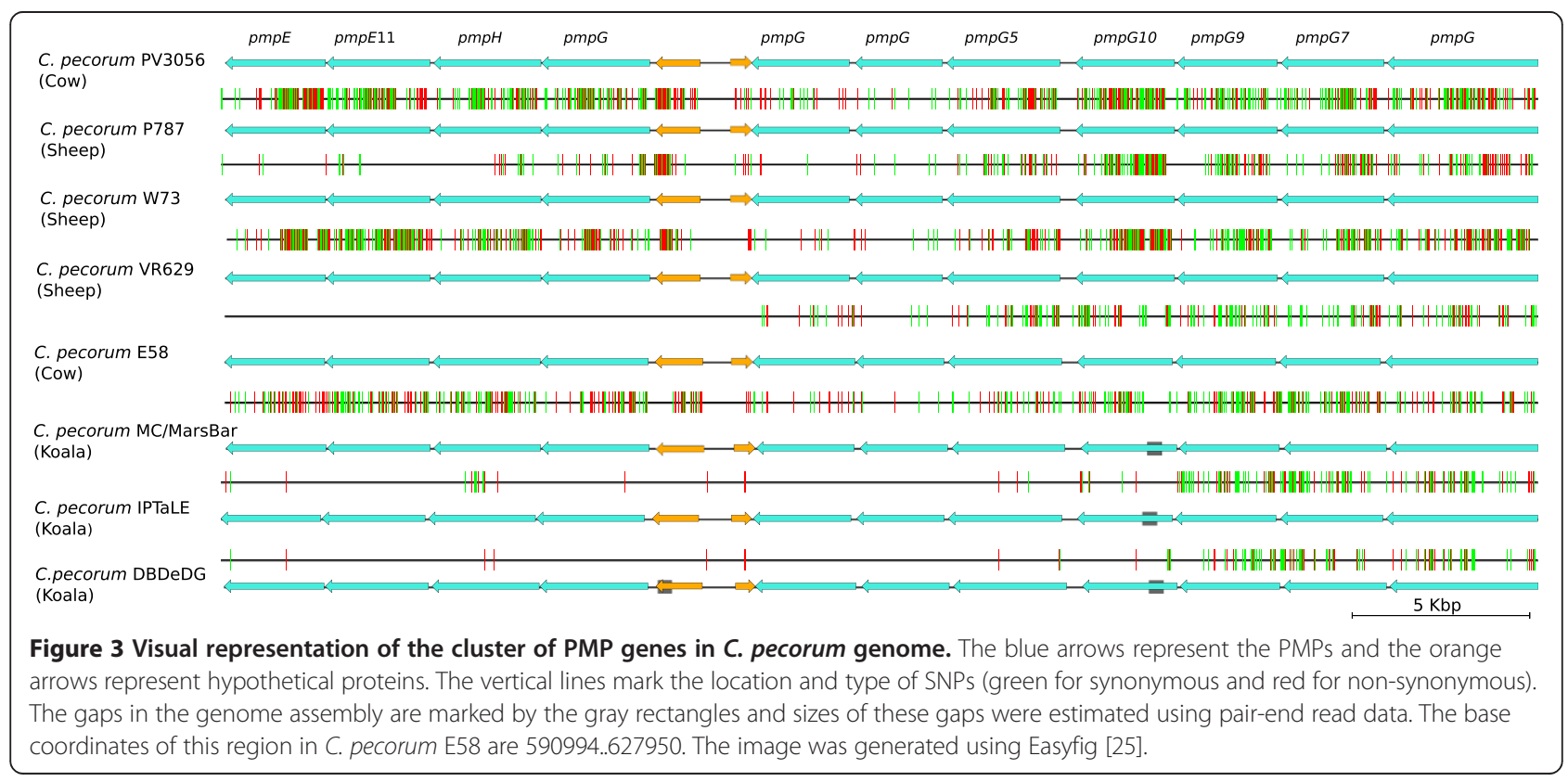




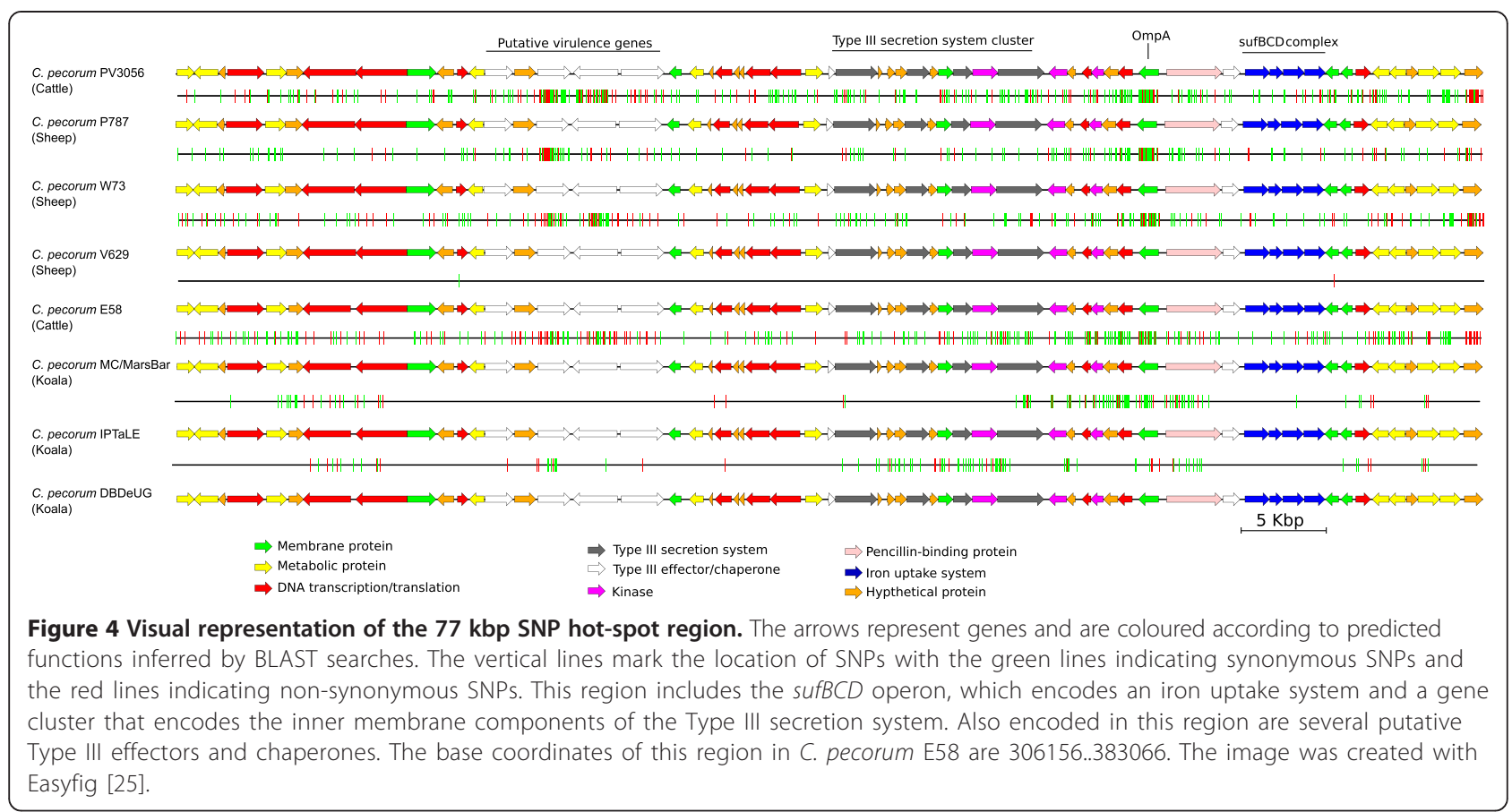

3' end [21]. The PZ in koala C. pecorum strains encodes two cytotoxin genes in tandem, in this study designated as tox $\mathrm{A}$ and tox $\mathrm{B}$. The $\mathrm{PZ}$ also contains several other genes that encode proteins linked to pathogenesis, including the MAC/perforin domain protein and five phospholipase D (PLD) [30,31]. The presence of two cytotoxic genes make the $C$. pecorum $\mathrm{PZ}$ unique compared to the PZ from other Chlamydia species. Although of similar size, the tox $\mathrm{A}$ and tox $\mathrm{B}$ genes share only $43 \%$ sequence identity on the amino acid level with several conserved motifs. The $\mathrm{N}$-terminal regions of the
toxA/B genes contain a catalytic glycosyltransferase domain. Interestingly, in the genome of C. pecorum $\mathrm{MC} /$ MarsBar, a SNP (C-T) in the toxB gene resulted in a premature stop codon at position 6440 , truncating the gene. The presence of the premature stop codon was confirmed with PCR amplification and sequencing of the toxB gene in the C. pecorum MC/MarsBar strain, as well as the other two koala and a sheep strain. The tox $\mathrm{B}$ gene is only truncated in C. pecorum MC/MarsBar and fulllength homologs are present in the other four C. pecorum genomes.

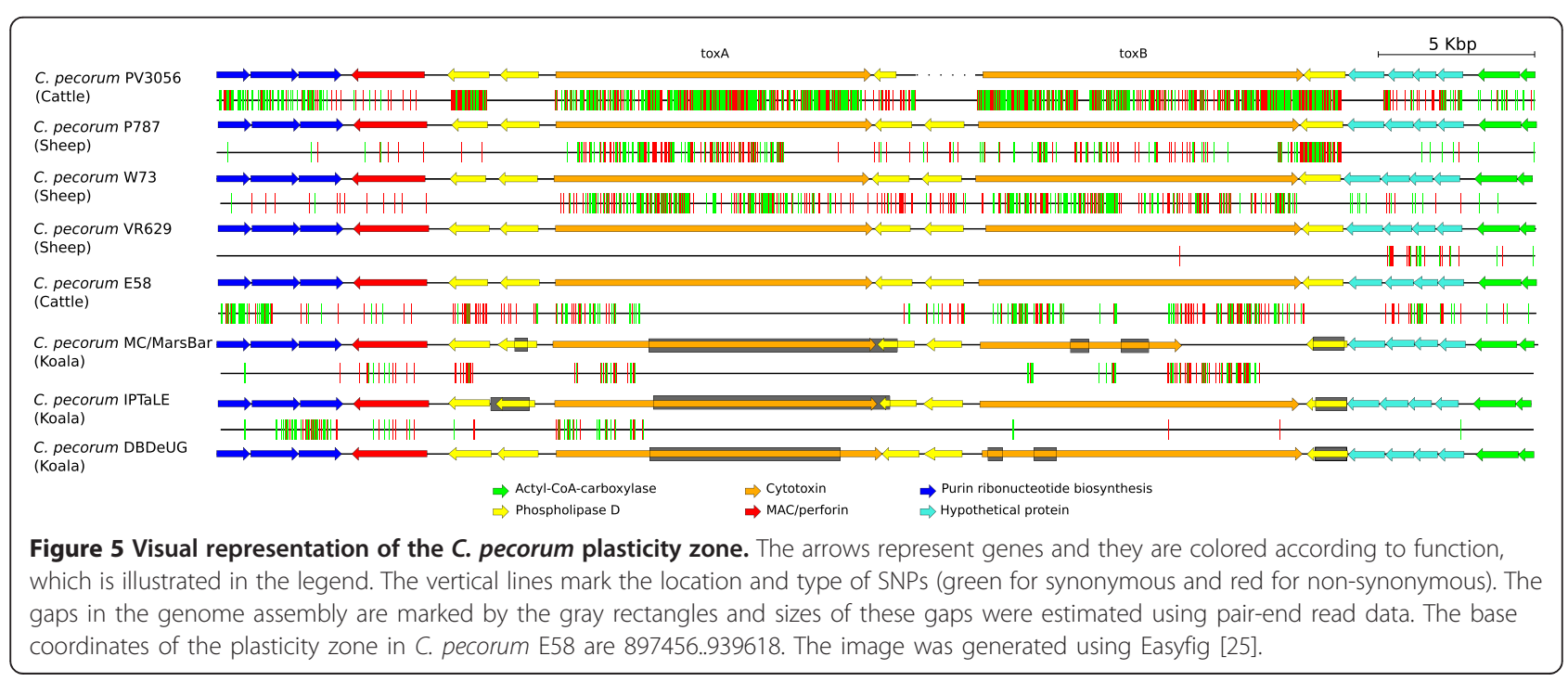


The presence of a limited number of pseudogenes further highlights genetic variation between koala $C$. pecorum strains

In addition to tox $B$, another five pseudogenes were identified in the three koala $C$. pecorum genomes, providing additional evidence of genetic diversity between C. pecorum strains isolated from koalas (Table 3 ). The pseudogenes in $C$. pecorum were caused by premature stop codons within the gene sequences as a result of SNPs. C. pecorum IPTaLE has three pseudogenes, one being the pyrE gene (CpecA_0392) that codes for an orotate phosphoribosyltransferase. The functions of the other two pseudogenes in C. pecorum IPTaLE are unknown, although one of the genes (CpecA_0640) contains a signal peptide at the $\mathrm{N}$-terminus suggesting that the encoded protein targets a secretory pathway. The genomes of $C$. pecorum MC/MarsBar and $C$. pecorum DBDeUG each have a single pseudogene that contains transmembrane domains; however their actual functions are unknown. All six pseudogenes, including to $x$, identified in the koala $C$. pecorum genomes are intact in the all the livestock $C$. pecorum genomes.

\section{Some but not all predicted koala $C$. pecorum pseudogenes are widely distributed in $C$. pecorum strains detected from across the koala's host range}

In a preliminary investigation into the broader genetic diversity of $C$. pecorum in Australian animals, we PCR amplified and sequenced partial regions of the six pseudogenes identified in the koala $C$. pecorum genomes from 73 C. pecorum PCR positive samples collected from the (a) conjunctival and urogenital tract sinuses of koalas from populations in Queensland, New South Wales, Victoria and South Australia $(n=65)$; and (b) conjunctiva and rectums of Australian sheep $(n=8)$ (see Additional file 4). The list of $C$. pecorum strains chosen was based on PCR-positive swab samples that have sufficient chlamydial DNA for multiple PCR amplification and to ensure an even distribution of strains from each Australian state. Out of the six pseudogenes identified from the genome sequences, four were also found to be pseudogenes in the clinical samples. The tox $B$ gene was found to be truncated in some $(n=11)$ but not all of the koala C. pecorum positive samples from populations in South-East Queensland, South Australia and Victoria. Indeed, we were able to identify 11 unique tox $B$ gene fragment sequences amongst the collection examined, with sequences varying in similarity between $84.3-99.7 \%$ across the livestock and koala samples (see Additional file 5). Similarly, one of the pseudogenes (CpecA_0641) from $C$. pecorum IPTaLE was also truncated in three urogenital $C$. pecorum positive swabs from three different koala hosts, however the truncation is caused by a different mutation than in $C$. pecorum IPTaLE. The pseudogene (CpecG_0412) from C. pecorum MC/MarsBar was found to be truncated in another two $C$. pecorum positive samples collected from the cloaca and urethra of a koala in Victoria and NSW, respectively. The pseudogene (CpecF_0874) from C. pecorum DBDeUG was also confirmed to be a pseudogene in seven $C$. pecorum positive samples from the conjunctiva and genital tracts of koalas in Queensland only. Interestingly, the remaining two pseudogenes (pyrE and CpecA_0640) from C. pecorum IPTaLE were intact in all koala $C$. pecorum samples analysed. Although only a smaller sample set was analyzed, the evidence that all pseudogenes identified and confirmed in the koala C. pecorum genomes and clinical samples appeared to be intact in Australian livestock was notable. While this observation appeared to further distinguish koala from livestock $C$. pecorum strains, it is also worth noting that a number of the new partial gene sequences identified in this analysis for each of these marker genes were otherwise identical (see Additional file 5). This was confirmed with a phylogenetic tree based on the concatenated sequences of five of the six pseudogenes pyrE gene sequences were difficult to amplify for a number of samples - not showing any clear distinction between koala and livestock strains from Europe, the USA and Australia (Figure 6).

\section{Discussion}

While the initial sequencing of chlamydial genomes has provided important insights into the general biology of the chlamydiae, reconciling the minor genetic differences between different strains of the same species with particular diseases, hosts and sites of infection has been

Table 3 Pseudogenes caused by premature stop codons in C. pecorum strains isolated from koalas

\begin{tabular}{llll}
\hline Strain & Locus tag & Protein description & Percentage of gene truncated \\
\hline C. pecorum IPTaLE & CpecA_0392 & Orotate phosphoribosyltransferase & $64 \%$ \\
& CpecA_0639 & Hypothetical protein & $38 \%$ \\
& CpecA_0640 & Hypothetical protein & $24 \%$ \\
C. pecorum MC/MarsBar & CpecG_0412 & Hypothetical protein & $19 \%$ \\
C. pecorum DBDeUG & CpecG_0814 & Cytotoxin & $37 \%$ \\
\hline
\end{tabular}




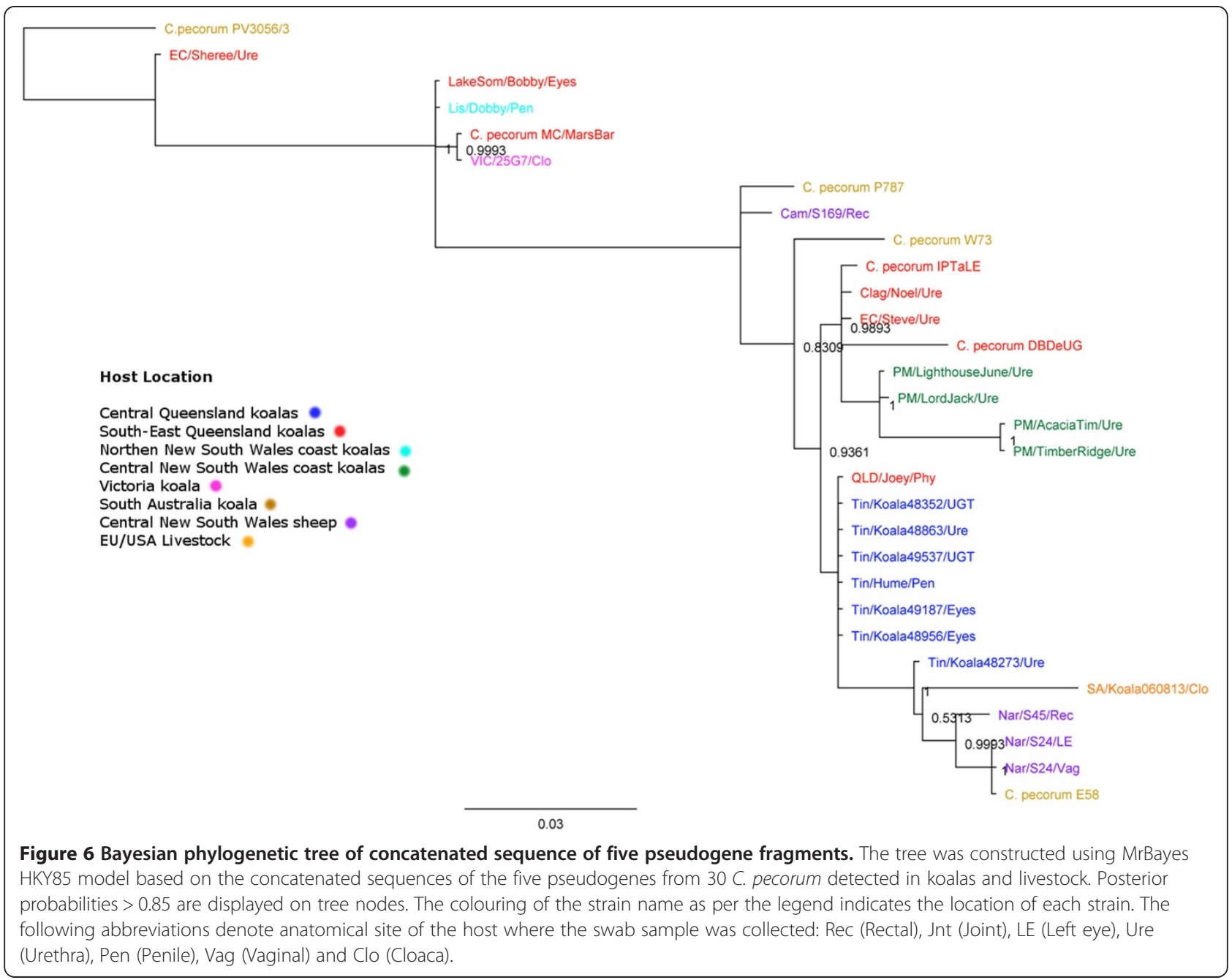

more challenging. C. pecorum is a useful model to investigate adaptation to different niches since it is a major pathogen with a broad host range and tissue tropisms [11-13]. Like most Chlamydia species, C. pecorum genomes are conserved in terms of gene content and order [21]. This high degree of gene conservation is believed to be the result of Chlamydia's intracellular lifestyle, which reduces exposure to frequent lateral gene transfer events that often cause phenotypic changes in other bacterial genera [32]. Therefore, small variations in the C. pecorum genomes are the likely causes for different phenotypic traits that are observed in different strains.

When it comes to investigating the genetic diversity of C. pecorum, one of the limiting factors has been the lack of genome sequences for $C$. pecorum strains isolated from different animals, in particular strains isolated from koalas. In this study we examined the draft genomes of three C. pecorum strains that were isolated from koalas in South-East Queensland, Australia. Each of the three koala C. pecorum genomes represents a different genotype, based on allelic differences in the ompA gene. Also sequenced, as part of this study, is the genome of C. pecorum VR629 (IPA), a strain that was isolated from the joint of a sheep suffering polyarthritis in the USA. The C. pecorum VR629 genome along with the four publically available C. pecorum genomes serve as references for comparison to the koala $C$. pecorum genomes, to assess the level of genetic diversity between $C$. pecorum strains [21,24]. The PZ, the PMP gene cluster and the $77 \mathrm{kbp}$ region near the origin of replication are hotspots for SNPs accumulation in the Australian koala strains and the European livestock strains. A similar high degree of SNPs is also observed in these same regions in $C$. trachomatis genomes (see Additional file 6). The gene content of the PZ and the $77 \mathrm{kbp}$ region were also compared between C. trachomatis and C. pecorum using discontinuous megablast [33]. While the gene content of the PZ is significantly different between $C$. pecorum and C. trachomatis, the only difference in the $77 \mathrm{kbp}$ hotspot region between both species is that the putative virulence genes in 
C. pecorum are absent in C. trachomatis. The $\mathrm{PZ}$ and the $77 \mathrm{kbp}$ region are highly conserved between the two American $C$. pecorum strains but additional genome sequencing of isolates from the USA is needed to confirm if this level of conservation is unique to USA strains.

Identification of SNPs between $C$. pecorum genomes from livestock and koalas has provided insight into which genes are likely to be involved in host adaptation. The most rapidly evolving genes in C. pecorum are the genes coding for the PMPs, a family of proteins that may be important for adhesion of Chlamydia to host cells $[4,34,35]$. In particular, the pmpG subfamily represents the most variable class of PMPs in C. pecorum, which is also the most rapidly evolving PMP class in C. psittaci [6]. Despite the variation in nucleotide sequences, the members of the PMP family possess a conserved domain structure that includes the C-terminal autotransporter beta-domain, a central domain unique to this family of proteins and a N-terminal domain that is involved in adhesion [36]. PMPs share sequence similarity to the autotransporter class of proteins, which have a diverse array of functions and often play an essential role in pathogenesis, thus the observed variation could also contribute to immune evasion [37].

This study has identified several novel putative virulence genes that could be specifically involved with adaptation to different hosts. These virulence genes include a putative surface protein SrpA1 and two putative Type III effectors. Type III effectors are virulence factors often involved in interactions with host cellular proteins in order to enhance the survival of the bacteria [38]. Amino acid changes introduced by non-synonymous SNPs could alter the binding domains of the effectors, altering their affinity to cellular targets. The other potential virulence factor of interest is the putative surface protein SrpA1; the observed variation in the protein sequence could alter binding and/or recognition to surface receptors of host cells, however, experimental confirmation is obviously required for further investigations. However, the accumulation of non-synonymous SNPs in srpA1 and the two putative effector genes could also be due to a selective pressure for avoiding antibody and cellular immune responses.

Our comparison of the $C$. pecorum genomes revealed the presence of six pseudogenes in the strains isolated from koalas, one of these pseudogenes being the tox $B$ gene located in the $\mathrm{PZ}$ in $C$. pecorum MC/Marsbar. C. pecorum typically possesses two full-length cytotoxin genes in the $\mathrm{PZ}$, designated tox $\mathrm{A}$ and tox $\mathrm{B}$. The full-length Chlamydia cytotoxin is similar to the large clostridial cytotoxin (LCTs) from Clostridium difficile, which also has the glycosyltransferase domain in the $\mathrm{N}$-terminal region of the protein. This domain has been shown to interfere with eukaryotic cells by glycosylating
GTP binding proteins of the Ras superfamily, inactivating them and leading to disassembly of the actin cytoskeleton $[39,40]$. The LCTs also include a domain for binding to the surface receptors of eukaryotic cells and a transmembrane segment that is involved in translocation into the cytoplasm [41]. C. pecorum MC/MarsBar has a full-length toxA gene and a shorter tox $\mathrm{B}$ gene that has been truncated by a premature stop codon. The truncation of tox $\mathrm{B}$ was observed in 11 of the 65 C. pecorum positive swabs samples for which partial gene sequences could be amplified from wild koala, suggesting that it could be a recent mutation since the remaining sequences identified were otherwise intact. It is plausible that the C-terminal domain of the tox $\mathrm{B}$ gene is being deleted to reduce the energetic cost of maintaining the full-length gene [42].

Out of the other five pseudogenes in the koala $C$. pecorum genomes, only the pyrE gene has a predicted function. The gene pyrE encodes an orotate phosphoribosyltransferase, an enzyme that is part of the de novo pyrimidine biosynthesis pathway [43]. Chlamydia spp. cannot synthesise pyrimidine nucleotides de novo as key genes involved in this pathway are missing [44], but instead must rely on a salvaging pathway that involves transporting ribonucleotides directly from the host cell [45]. The presence of the pyrE gene could be a remnant from when Chlamydia was able to synthesise pyrimidine de novo; this is supported by the observed fragmentation of pyrE in $C$. pecorum IPTaLE, indicating gene decay. Interestingly, despite this potentially important observation, the pyrE gene was found to be intact in all koala C. pecorum strains that were screened, raising questions over whether this SNP may have resolved during laboratory passage. In addition to this pseudogene, there are two other pseudogenes that are unique to the C. pecorum IPTaLE conjunctival isolate, compared to the sequenced urogenital DBDeUG and MC/Marsbar strains. However, while one of these pseudogenes was found to be intact in all samples tested, the other (CpecA_0641) was confirmed to be a pseudogene in three koala urogenital samples from the same geographic region and therefore both of these genes are unlikely to be involved in tissue tropism.

Sequence alignments of the pseudogene fragments revealed that some genotypes are specific to $C$. pecorum detected in Australian livestock and koala hosts (see Additional file 5). However, a phylogenetic analysis of the concatenated pseudogene sequences showed that, collectively, there was no host specific separation in contrast to our whole genome phylogenies (Figure 6). The observation that (a) multiple $C$. pecorum genotypes can be circulating in a single population of animals; and (b) individual genotypes can be found across multiple populations of koalas or livestock is otherwise consistent with our previous fine-detailed molecular epidemiological investigations $[15,18,19]$. 


\section{Conclusions}

While the primary focus of this study was to examine the genetic differences potentially associated with C. pecorum host adaptation, the observed accumulation of genetic changes in koala $C$. pecorum strains provides an opportunity to speculate on the origin of this pathogen in the koala. The koala C. pecorum genomes contain a small number of pseudogenes and gene truncations, which are otherwise intact in the livestock genomes. These mutations suggest that C. pecorum is evolving to adapt to the koala through the loss of genes that are no longer necessary. Although our genome phylogenies do not yet support this, the most parsimonious explanation for this observation is that koala C. pecorum strains have derived from livestock strains and are undergoing genetic changes to better adapt to the new host. Interestingly enough, if this is true, then it would be the reverse of what we have previously observed for koala Chlamydia pneumoniae, whereby the genome of the koala strain was found to be largely intact compared to human strains, suggesting that the koala strains were ancestral [44]. In order to validate that koala C. pecorum strains are indeed derived from livestock strains it is necessary to reconstruct the evolutionary history of C. pecorum using phylogenetic and molecular clock analyses. However, additional C. pecorum strains will need to be sequenced in order to fill in the missing links from this comparison including analysis of C. pecorum isolates from Australian livestock. Indeed, a limitation of the phylogenetic analysis performed in this study is the small number of available C. pecorum genomes. C. pecorum PV3056 was expected to cluster together with C. pecorum W37 and C. pecorum P787 based on the geographic location of these strains but the PV3056 strain is phylogenetically distinct from the other C. pecorum strains. Therefore, it is important to sequence more $C$. pecorum strains in order to fully understand the phylogenetic structure of this species but also to learn more about the role of the observed limited gene variation in disease pathogenesis and tissue and host adaptation.

\section{Methods}

Bacterial strains, chlamydial cell culturing and enrichment for genome sequencing

Three koala C. pecorum strains utilised for genome sequencing and comparative genomics were propagated in our laboratory. Swab samples were collected from three wild koalas residing in South East Queensland, Australia and stored in SPG transport media [46]. C. pecorum DBDeUG (QLD/SEF/UGT) strain was isolated from the urogenital tract of a wild female koala suffering from a urogenital tract infection, while the C. pecorum IPTaLE (QLD/IpsA/Eye) strain was isolated from the left conjunctiva of a wild male koala suffering from conjunctivitis.
C. pecorum MC/MarsBar strain, previously described by Marsh et al. [19], was isolated from a female koala suffering from severe ocular and urogenital tract disease. The collection of these swabs by qualified veterinarians as a part of routine diagnostic testing and the subsequent Chlamydia culturing has been considered by the Queensland University of Technology (QUT) Animal Ethics Committee and approved as Tissue Use Notification \# 1100000718. We also sequenced the genome of the ovine C. pecorum polyarthritis strain IPA (ATCC VR629), originally isolated from the joint fluid of a sheep in Iowa, USA [47]. In addition, we also made use of the full genome sequence of C. pecorum E58, an isolate that was collected from the brain of a calf suffering sporadic bovine encephalomyelitis [24]. Also included are the complete genomes of $C$. pecorum W73, C. pecorum P787 and C. pecorum PV3056, which were sequenced from strains infecting livestock animals in Europe [21].

The koala C. pecorum isolates were individually propagated in Hep-2 cells while the ovine C. pecorum isolate was propagated in McCoy cells. Following density gradient centrifugation, ultrapurified EBs for each strain were treated with DNAase and then purified using a QIAamp DNA Mini Kit (Qiagen), according to the manufacturer's instructions, followed by repeated sodium acetate/ethanol precipitation and pellet resuspension in $0.1 \mathrm{M}$ TE buffer.

\section{Prospective screening of koala samples using C. pecorum-specific PCR screen}

To expand the analysis of C. pecorum strains from koalas outside of South-East Queensland, conventional PCR-based C. pecorum specific screening was performed on a range of koala samples collected from koala populations in Queensland, South Australia and New South Wales. From these regions, a total of 156 swabs were collected from 62 koalas presenting for treatment at the Australia Zoo Wildlife Hospital $(\mathrm{n}=32)$, Adelaide Hills Animal Hospital $(n=23)$, and Port Macquarie Koala Hospital $(\mathrm{n}=13)$, respectively. For each animal, sampling included a collection of conjunctival and urogenital sinus samples, while nasal samples were also collected from the South Australian koalas. In addition to the prospectively screened samples, a collection of 35 previously analysed C. pecorum positive samples were included in this analysis including samples from (i) various koala populations in South-East Queensland $(\mathrm{n}=20)$, New South Wales ( $\mathrm{n}=6)$ and Victoria ( $\mathrm{n}=1)$; and (ii) Australian livestock sampled in Central New South Wales $(n=8)$ $[18,19]$. Prospectively collected samples were screened for the presence of $C$. pecorum DNA using a C. pecorum specific qPCR assay, that targets a 202 base pair region of the C. pecorum $16 \mathrm{~S}$ rRNA gene, as previously described [48] using extracted DNA as a template. 


\section{Genome sequencing}

The genomes of the four C. pecorum strains were sequenced using Illumina HiSeq to produce, paired-end 100 base-pair reads. Read quality was checked with FASTQC and filtering was performed on the reads with PrinSeq-Lite to ensure a mean base-pair quality of score greater than 20 . The paired-end reads were randomly selected so that coverage of $100 \times$ was achieved for each genome. The genomes were assembled de novo using SOAPdenovo with an optimal $k$-mer of 33, which was determined by individually testing odd $k$-mer values ranging from 25 to 35 . The genomes were further assembled into a single scaffold using GapCloser [49]. C. pecorum VR629 (ATCC IPA) was assembled into four contigs, while the genomes for the three koala C. pecorum strains (C. pecorum DBDeUG (QLD/ SEF/UGT), C. pecorum IPTaLE (QLD/IpsA/Eye) and C. pecorum $\mathrm{MC} / \mathrm{MarsBar}$ ) were assembled into contigs ranging from 8 to 13 contigs. The contigs for each genome were ordered against the complete genome of $C$. pecorum E58 (accession number: CP002608) [24]. The average N50 contig size is 479,694 base pairs and the average size of the assembled genomes is 1.1 megabase pairs. The four draft $C$. pecorum genomes were automatically annotated using GenDB [50]. The genome sequences for the three koala strains and the sheep were deposited in Genbank under accession numbers AZBE01000000 for C. pecorum IPTaLE, AZBB01000000 for C. pecorum DBDeUG, AZBC01000000 for C. pecorum MC/Marsbar and AZBD01000000 for C. pecorum VR629.

\section{Phylogenetic analyses and genome comparison}

The following genome sequences were used in comparative and phylogenetic analyses with the four C. pecorum genomes sequenced in this study: $C$. muridarum NIGG (accession number: AE002160), C. trachomatis A/HAR-13 (accession number: CP000051), C. trachomatis L2/434/Bu (accession number: AM884176), C. caviae (accession number: AE015925), C. felis Fe/C-56 (accession number: AP006861), C. abortus S26/3 (accession number: CR848038), C. psittaci 6BC (accession number: CP002549), C. pneumoniae AR39 (accession number: AE002161), C. pneumoniae LPCoLN (accession number: CP001713), C. pecorum E58 (accession number: CP002608), C. pecorum P787 (accession number: CP04035), C. pecorum W73 (accession number: CP004034) and C. pecorum PV3056/3 (accession number: CP04033).

A phylogenetic analysis was performed using 152 orthologous genes with $>70 \%$ nucleotide identity and $<20 \%$ difference in gene length from the 14 Chlamydia genomes, which includes representatives from all major species. The nucleotide sequences of the 152 genes were extracted from each genome and individually aligned using MUSCLE [51], and then concatenated. A phylogenetic tree was constructed by the maximum-likelihood method using the General Time Reversible (GTR) model with PhyML 3.0 [52]. Bootstrap values were calculated using 500 replicates.

Pairwise whole genome comparisons of the four draft C. pecorum genomes were performed using BLASTn and visualised with the Artemis Comparison Tool [53]. Figures of the whole genome comparison were generated using BRIG (BLAST Ring Image Generator) [54] and Easyfig [25]. Selection analysis was performed with KaKs Calculator 2.0 using the LWL model $[29,55]$. The selective pressure acting on genes can be measured by calculating the ratio of non-synonymous to synonymous substitutions $\left(d_{n} / d_{s}\right)$. Low $d_{n} / d_{s}$ values (less than 1 ) are indicative of purifying selection, which means that the gene is being maintained and most of the substitutions are synonymous. On the other hand, high $d_{n} / d_{s}$ ratios (greater than 1) are usually suggestive of positive selection in which the gene is accumulating non-synonymous substitutions that result in changes in the amino acid sequence of the encoded protein [56].

\section{SNP prediction}

The filtered reads of each C. pecorum strain were mapped individually against the genome of $C$. pecorum E58 acquired with $12 \times$ coverage (Assembly ID: GCF_ 000204135.1) and the assembled scaffolds of the other C. pecorum genomes using the BWA-backtrack algorithm with BWA aligner [57]. The BWA parameters used include the number of differences allowed between the reference and query set at 0.04 and the number of differences allowed in the seed was 2 . The maximum number of gaps allowed in the alignment was 1 and the gap penalty was set at 11 . SNPs were predicted using the variant caller program, VarScan with default settings [58]. Progressive Mauve with default setting was used to align the complete $C$. pecorum genomes and call SNPs since the reads were not available [59]. A custom Perl script was used to determine if a SNP caused a synonymous change, non-synonymous change or a change that introduce premature stop codons within CDSs leading to the formation of pseudogenes. A custom $\mathrm{R}$ script was used to plot the distribution of SNPs across the genome with a window size of $100 \mathrm{kbp}$. Predicted SNPs within the PZ, PMP gene cluster and the $77 \mathrm{kbp}$ hypervariable region as well as within the six potential pseudogenes were manually and visually inspected using the BAM files generated from the BWA read mapping in Artemis.

\section{Targeted PCR amplification of predicted $C$. pecorum} pseudogenes in koala and livestock $C$. pecorum strains In order to confirm the truncation of each pseudogene and to survey a broader selection of $C$. pecorum samples 
from koalas and Australian livestock, C. pecorum specific primers were designed for PCR amplification of a 250$500 \mathrm{bp}$ region of each gene, which includes the predicted stop codons observed in our genome analyses (see Additional file 7). Each PCR assay was prepared to a total volume of $50 \mu \mathrm{l}$, consisting of 1 X Amplitaq Gold 360 Mastermix (Life Technologies, Victoria, AUS), $0.3 \mu \mathrm{M}$ of Forward and Reverse primers (Sigma-Aldrich, New South Wales, AUS) and $3 \mu \mathrm{l}$ DNA template, of an average concentration of $30 \mathrm{ng} / \mu \mathrm{l}$. The cycling conditions for the PCR amplification of the six pseudogene fragments included initial denaturation $\left(10 \mathrm{~min}, 95^{\circ} \mathrm{C}\right)$ followed by 40 cycles of denaturation $\left(30 \mathrm{~s}, 95^{\circ} \mathrm{C}\right)$, annealing (tox $\mathrm{B}$ $30 \mathrm{~s}, \quad 61.5^{\circ} \mathrm{C}$; pyrE $30 \mathrm{~s}, 56^{\circ} \mathrm{C}$; C.pecA_0641 30s, $53^{\circ} \mathrm{C}$; CpecA_0640 and CpecG_0412 30s, $49^{\circ} \mathrm{C}$; and CpecF_0874 $\left.30 \mathrm{~s}, 58.6^{\circ} \mathrm{C}\right)$ and extension $\left(1 \mathrm{~min}, 72^{\circ} \mathrm{C}\right)$, followed by a final extension $\left(7 \mathrm{~min}, 72^{\circ} \mathrm{C}\right)$. Negative controls $\left(\mathrm{dH}_{2} \mathrm{O}\right.$ and no template) were included in each amplification assay. Primer characteristics are outlined in Additional file 7.

Each PCR product was directly sequenced using a BigDye Terminator v3.1 Cycle Sequencing kit (Life Technologies, Victoria, Australia) and subsequently purified according to the manufacturer's instructions. Sequencing was performed at the Queensland University of Technology DNA sequencing facilities, using the Applied Biosystems ABI3500 Gene analyser. The acquired sequences of the livestock and koala $C$. pecorum fragments for each gene were aligned using ClustalW [60] and translated as implemented in Geneious Pro 7, in order to confirm the presence of the observed stop codon in each of the strains analysed. A phylogenetic tree based on the concatenation of five pseudogene fragment sequences was constructed using MrBayes [61]. The nucleotide sequences for each of the six pseudogene fragments amplified in this study from the $C$. pecorum positive samples are available in GenBank under the accession numbers KJ804269-KJ804400.

\section{Additional files}

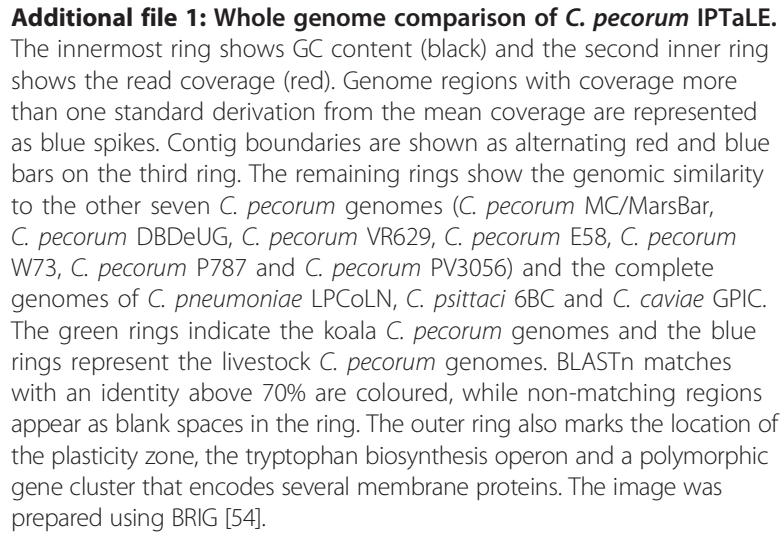

Additional file 2: List of genes in the 77-kbp SNP hotspot region. A table of genes in the 77-kbp SNP hotspot regions from C. pecorum MC/ MarsBar. The number of SNPs and ka/ks ratio is based on the comparison of these genes between C. pecorum MC/MarsBar and C. pecorum E58.

Additional file 3: Alignment of the surface anchor protein SrpA1 from $C$. pecorum. The multiple protein alignment shows the full length of the SrpA1 surface protein from C. pecorum IPTaLE, C. pecorum MC/ MarsBar, C. pecorum DBDeUG, C. pecorum VR629 and C. pecorum E58. The alignment is coloured using the BLOSUM62 scoring matrix. The C-terminal domain is conserved in all five C. pecorum genomes but the N-terminal part of the protein contains significant variation.

Additional file 4: Summary table of PCR screening for pseudogenes in $C$. pecorum positive samples. The table shows the status of the six pseudogenes in 65 C. pecorum positive swab samples from koalas and sheep and in the seven available C. pecorum genome sequences.

Additional file 5: Nucleotide alignment of pseudogenes fragments amplified with PCR. Nucleotide alignments of the different alleles of the six pseudogenes fragments identified in a PCR screening of 65 C. pecorum strains.

Additional file 6: SNP distribution in C. trachomatis genomes. The histograms show the number of SNPs in relation to the genomic positions between different $C$. trachomatis genomes with a window size of $100 \mathrm{~kb}$. The top graph shows the SNP distribution between C. trachomatis D/UW-3/CX (accession number: AE001273) and C. trachomatis A/HAR-13 (accession number: CP00051). The bottom graph shows the SNP distribution between C. trachomatis D/UW-3/CX and C. trachomatis L2/434/Bu (accession number: AM884176). The red boxes mark the SNP hotspot regions that were also observed in C. pecorum.

Additional file 7: Table of primers used in this study. Contains the list of primers used to amplify the six pseudogenes.

\section{Competing interests}

The authors declare that they have no competing interests.

\section{Authors' contributions}

The genomic analyses were carried out by NLB and CB. PCR screening and DNA sequencing and analyses was performed by TF and MJ. Koala swab samples were collected by AG, OF and CF. Genome sequencing was carried out by GASM. The study was conceived and designed by AP and PT. All authors have read and approved the final manuscript.

\section{Acknowledgements}

This work was financially supported by an Australian Research Council Discovery Grant (DP130102066) and a Queensland Department of Environment and Heritage Protection Koala Research Grant awarded to AP and PT. We would like to thank Alexander Goesmann, Centre for Biotechnology, Universität Bielefeld for access to the GenDB annotation system.

\section{Author details}

${ }^{1}$ Faculty of Science, Health, Education and Engineering, University of the Sunshine Coast, Sippy Downs 4558, Queensland, Australia. ${ }^{2}$ Institute for Health and Biomedical Innovation, Queensland University of Technology, Kelvin Grove 4059, Queensland, Australia. ${ }^{3}$ SIB Swiss Institute of Bioinformatics, Lausanne, Switzerland. ${ }^{4}$ Australia Zoo Wildlife Hospital, Beerwah, Queensland 4519, Australia. ${ }^{5}$ Adelaide Hills Animal Hospital, Stirling, South Australia 5152, Australia. ${ }^{6}$ Port Macquarie Koala Hospital, Port Macquarie, NSW 2444, Australia. ${ }^{7}$ Institute for Genome Sciences, University of Maryland School of Medicine, Baltimore 21201, USA. ${ }^{8}$ Current address: Center for Research on Intracellular Bacteria, Institute of Microbiology, University Hospital Center and University of Lausanne, Lausanne, Switzerland.

Received: 24 May 2014 Accepted: 31 July 2014

Published: 8 August 2014

\section{References}

1. Hogan RJ, Mathews SA, Mukhopadhyay S, Summersgill JT, Timms P: Chlamydial persistence: beyond the biphasic paradigm. Infect Immun 2004, 72(4):1843-1855. 
2. Verminnen K, Duquenne B, De Keukeleire D, Duim B, Pannekoek Y, Braeckman L, Vanrompay D: Evaluation of a Chlamydophila psittaci infection diagnostic platform for zoonotic risk assessment. J Clin Microbiol 2008, 46(1):281-285.

3. Anderson IE, Baxter SI, Dunbar S, Rae AG, Philips HL, Clarkson MJ, Herring AJ: Analyses of the genomes of chlamydial isolates from ruminants and pigs support the adoption of the new species Chlamydia pecorum. Int I Syst Evol Microbiol 1996, 46(1):245-251.

4. Thomson NR, Yeats C, Bell K, Holden MT, Bentley SD, Livingstone M, Cerdeno-Tarraga AM, Harris B, Doggett J, Ormond D, Mungall K, Clarke K, Feltwell T, Hance Z, Sanders M, Quail MA, Price C, Barrell BG, Parkhill J, Longbottom D: The Chlamydophila abortus genome sequence reveals an array of variable proteins that contribute to interspecies variation. Genome Res 2005, 15(5):629-640.

5. Bodetti TJ, Jacobson E, Wan C, Hafner L, Pospischil A, Rose K, Timms P: Molecular evidence to support the expansion of the hostrange of Chlamydophila pneumoniae to include reptiles as well as humans, horses, koalas and amphibians. Syst App Microbiol 2002, 25(1):146-152.

6. Voigt A, Schofl G, Saluz HP: The Chlamydia psittaci genome: a comparative analysis of intracellular pathogens. PLoS One 2012, 7(4):e35097.

7. Harris SR, Clarke IN, Seth-Smith HM, Solomon AW, Cutcliffe LT, Marsh P, Skilton RJ, Holland MJ, Mabey D, Peeling RW, Lewis DA, Spratt BG, Unemo M, Persson K, Bjartling C, Brunham R, de Vries HJ, Morre SA, Speksnijder A, Bebear CM, Clerc M, de Barbeyrac B, Parkhill J, Thomson NR: Whole-genome analysis of diverse Chlamydia trachomatis strains identifies phylogenetic relationships masked by current clinical typing. Nat Genet 2012, 44(4):413-419. S411.

8. Fraser C, Hanage WP, Spratt BG: Neutral microepidemic evolution of bacterial pathogens. Proc Natl Acad Sci U S A 2005, 102(6):1968-1973.

9. Bush RM: Predicting adaptive evolution. Nat Rev Genet 2001, 2(5):387-392.

10. Borges V, Nunes A, Ferreira R, Borrego MJ, Gomes JP: Directional evolution of Chlamydia trachomatis towards niche-specific adaptation. J Bacteriol 2012, 194(22):6143-6153

11. Fukushi H, Hirai K: Proposal of Chlamydia pecorum sp. nov. for Chlamydia strains derived from ruminants. Int J Syst Evol Microbiol 1992, 42(2):306-308.

12. Polkinghorne $A$, Borel $N$, Becker $A$, Lu ZH, Zimmermann DR, Brugnera $E$, Pospischil A, Vaughan L: Molecular evidence for chlamydial infections in the eyes of sheep. Vet Microbiol 2009, 135(1-2):142-146.

13. Jee J, Degraves FJ, Kim T, Kaltenboeck B: High prevalence of natural Chlamydophila species infection in calves. J Clin Microbiol 2004, 42(12):5664-5672

14. Poudel A, Elsasser TH, Rahman Kh S, Chowdhury EU, Kaltenboeck B: Asymptomatic endemic Chlamydia pecorum infections reduce growth rates in calves by up to 48 percent. PLoS One 2012, 7(9):e44961.

15. Jackson M, White N, Giffard P, Timms P: Epizootiology of Chlamydia infections in two free-range koala populations. Vet Microbiol 1999, 65(4):255-264

16. McColl KA, Martin RW, Gleeson LJ, Handasyde KA, Lee AK: Chlamydia infection and infertility in the female koala (Phascolarctos cinereus). Vet Rec 1984, 115(25-26):655.

17. Polkinghorne A, Hanger J, Timms P: Recent advances in understanding the biology, epidemiology and control of chlamydial infections in koalas. Vet Microbiol 2013, 165(3-4):214-223.

18. Jelocnik M, Frentiu FD, Timms P, Polkinghorne A: Multi-locus sequence analysis provides insights into the molecular epidemiology of Chlamydia pecorum infections in Australian sheep, cattle and koalas. J Clin Microbiol 2013, 51(8):2625-2632

19. Marsh J, Kollipara A, Timms P, Polkinghorne A: Novel molecular markers of Chlamydia pecorum genetic diversity in the koala (Phascolarctos cinereus). BMC Microbiol 2011, 11:77.

20. Yousef Mohamad K, Roche SM, Myers G, Bavoil PM, Laroucau K, Magnino S, Laurent S, Rasschaert D, Rodolakis A: Preliminary phylogenetic identification of virulent Chlamydophila pecorum strains. Infect Genet Evol 2008, 8(6):764-771

21. Sait M, Livingstone M, Clark EM, Wheelhouse N, Spalding L, Markey B, Magnino S, Lainson FA, Myers GS, Longbottom D: Genome sequencing and comparative analysis of three Chlamydia pecorum strains associated with different pathogenic outcomes. BMC Genomics 2014, 15(1):23.

22. Kollipara A, Polkinghorne A, Wan C, Kanyoka P, Hanger J, Loader J, Callaghan J, Bell A, Ellis W, Fitzgibbon S, Melzer A, Beagley K, Timms P: Genetic diversity of Chlamydia pecorum strains in wild koala locations across Australia and the implications for a recombinant $C$. pecorum major outer membrane protein based vaccine. Vet Microbiol 2013, 167(3-4):513-522.

23. Stephens RS, Myers G, Eppinger M, Bavoil PM: Divergence without difference: phylogenetics and taxonomy of Chlamydia resolved. FEMS Immunol Med Microbiol 2009, 55(2):115-119.

24. Mojica S, Huot Creasy H, Daugherty S, Read TD, Kim T, Kaltenboeck B, Bavoil P, Myers GS: Genome sequence of the obligate intracellular animal pathogen Chlamydia pecorum E58. J Bacteriol 2011, 193(14):3690.

25. Sullivan MJ, Petty NK, Beatson SA: Easyfig: a genome comparison visualizer. Bioinformatics 2011, 27(7):1009-1010.

26. Fields KA, Mead DJ, Dooley CA, Hackstadt T: Chlamydia trachomatis type III secretion: evidence for a functional apparatus during earlycycle development. Mol Microbiol 2003, 48(3):671-683.

27. Hefty PS, Stephens RS: Chlamydial type III secretion system is encoded on ten operons preceded by sigma 70-like promoter elements. J Bacterio/ 2007, 189(1):198-206.

28. Outten FW, Wood MJ, Munoz FM, Storz G: The SufE protein and the SufBCD complex enhance SufS cysteine desulfurase activity as part of a sulfur transfer pathway for Fe-S cluster assembly in Escherichia coli. J Biol Chem 2003, 278(46):45713-45719.

29. Wang D, Zhang Y, Zhang Z, Zhu J, Yu J: KaKs_calculator 2.0: a toolkit incorporating gamma-series methods and sliding window strategies. Dev Reprod Biol 2010, 8(1):77-80.

30. Taylor LD, Nelson DE, Dorward DW, Whitmire WM, Caldwell HD: Biological characterization of Chlamydia trachomatis plasticity zone MACPF domain family protein CT153. Infect Immun 2010, 78(6):2691-2699.

31. Nelson DE, Crane DD, Taylor LD, Dorward DW, Goheen MM, Caldwell HD: Inhibition of chlamydiae by primary alcohols correlates with the strainspecific complement of plasticity zone phospholipase D genes. Infect Immun 2006, 74(1):73-80.

32. Toft C, Andersson SG: Evolutionary microbial genomics: insights into bacterial host adaptation. Nat Rev Genet 2010, 11(7):465-475.

33. Altschul SF, Gish W, Miller W, Myers EW, Lipman DJ: Basic local alignment search tool. J Mol Biol 1990, 215(3):403-410.

34. Read TD, Brunham RC, Shen C, Gill SR, Heidelberg JF, White O, Hickey EK, Peterson J, Utterback T, Berry K, Bass S, Linher K, Weidman J, Khouri H, Craven B, Bowman C, Dodson R, Gwinn M, Nelson W, DeBoy R, Kolonay J, McClarty G, Salzberg SL, Eisen J, Fraser CM: Genome sequences of Chlamydia trachomatis MoPn and Chlamydia pneumoniae AR39. Nucleic Acids Res 2000, 28(6):1397-1406.

35. Stephens RS, Lammel CJ: Chlamydia outer membrane protein discovery using genomics. Curr Opin Microbiol 2001, 4(1):16-20.

36. Wehrl W, Brinkmann V, Jungblut PR, Meyer TF, Szczepek AJ: From the inside out-processing of the Chlamydial autotransporter PmpD and its role in bacterial adhesion and activation of human host cells. Mol Microbiol 2004, 51(2):319-334

37. Henderson IR, Lam AC: Polymorphic proteins of Chlamydia spp.autotransporters beyond the Proteobacteria. Trends Microbiol 2001, 9(12):573-578.

38. Kleba B, Stephens RS: Chlamydial effector proteins localized to the host cell cytoplasmic compartment. Infect Immun 2008, 76(11):4842-4850.

39. Reinert DJ, Jank T, Aktories K, Schulz GE: Structural basis for the function of Clostridium difficile toxin B. J Mol Biol 2005, 351(5):973-981.

40. Busch C, Hofmann F, Selzer J, Munro S, Jeckel D, Aktories K: A common motif of eukaryotic glycosyltransferases is essential for the enzyme activity of large clostridial cytotoxins. J Biol Chem 1998, 273(31):19566-19572.

41. Richard JF, Petit L, Gibert M, Marvaud JC, Bouchaud C, Popoff MR: Bacterial toxins modifying the actin cytoskeleton. Int Microbiol 1999, 2(3):185-194.

42. Kuo $\mathrm{C}-\mathrm{H}$, Ochman $\mathrm{H}$ : The extinction dynamics of bacterial pseudogenes. PLoS Genet 2010, 6(8):e1001050.

43. Breda A, Rosado LA, Lorenzini DM, Basso LA, Santos DS: Molecular, kinetic and thermodynamic characterization of Mycobacterium tuberculosis orotate phosphoribosyltransferase. Mol Biosyst 2012, 8(2):572-586.

44. Mitchell CM, Hovis KM, Bavoil PM, Myers GS, Carrasco JA, Timms P: Comparison of koala LPCoLN and human strains of Chlamydia pneumoniae highlights extended genetic diversity in the species. BMC Genomics 2010, 11:442.

45. McClarty G, Qin B: Pyrimidine metabolism by intracellular Chlamydia psittaci. J Bacteriol 1993, 175(15):4652-4661. 
46. Warford AL, Rekrut KA, Levy RA, Drill AE: Sucrose phosphate glutamate for combined transport of chlamydial and viral specimens. Am J Clin Pathol 1984, 81(6):762-764.

47. Page LA, Cutlip RC: Chlamydia polyarthritis in lowa lambs. lowa Veterinarian 1968, 39:10-18.

48. Wan C, Loader J, Hanger J, Beagley K, Timms P, Polkinghorne A: Using quantitative polymerase chain reaction to correlate Chlamydia pecorum infectious load with ocular, urinary and reproductive tract disease in the koala (Phascolarctos cinereus). Aust Vet J 2011, 89(10):409-412.

49. Li R, Yu C, Li Y, Lam TW, Yiu SM, Kristiansen K, Wang J: SOAP2: an improved ultrafast tool for short read alignment. Bioinformatics 2009, 25(15):1966-1967.

50. Meyer F, Goesmann A, McHardy AC, Bartels D, Bekel T, Clausen J, Kalinowski J, Linke B, Rupp O, Giegerich R, Puhler A: GenDB-an open source genome annotation system for prokaryote genomes. Nucleic Acids Res 2003, 31(8):2187-2195.

51. Edgar RC: MUSCLE: multiple sequence alignment with high accuracy and high throughput. Nucleic Acids Res 2004, 32(5):1792-1797.

52. Guindon S, Delsuc F, Dufayard JF, Gascuel O: Estimating maximum likelihood phylogenies with PhyML. Methods Mol Biol 2009, 537:113-137.

53. Carver TJ, Rutherford KM, Berriman M, Rajandream MA, Barrell BG, Parkhill J: ACT: the Artemis Comparison Tool. Bioinformatics 2005, 21(16):3422-3423.

54. Alikhan NF, Petty NK, Ben Zakour NL, Beatson SA: BLAST Ring Image Generator (BRIG): simple prokaryote genome comparisons. BMC Genomics 2011, 12(1):402.

55. Li WH, Wu Cl, Luo CC: A new method for estimating synonymous and nonsynonymous rates of nucleotide substitution considering the relative likelihood of nucleotide and codon changes. Mol Biol Evol 1985, 2(2):150-174.

56. Hurst LD: The Ka/Ks ratio: diagnosing the form of sequence evolution. Trends Genet 2002, 18(9):486.

57. Li H, Durbin R: Fast and accurate short read alignment with BurrowsWheeler transform. Bioinformatics 2009, 25(14):1754-1760.

58. Koboldt DC, Chen K, Wylie T, Larson DE, McLellan MD, Mardis ER, Weinstock GM, Wilson RK, Ding L: VarScan: variant detection in massively parallel sequencing of individual and pooled samples. Bioinformatics 2009, 25(17):2283-2285.

59. Darling AE, Mau B, Perna NT: progressiveMauve: multiple genome alignment with gene gain, loss and rearrangement. PLoS One 2010, 5(6):e11147.

60. Larkin MA, Blackshields G, Brown NP, Chenna R, McGettigan PA, McWilliam H, Valentin F, Wallace IM, Wilm A, Lopez R, Thompson JD, Gibson TJ, Higgins DG: Clustal W and Clustal X version 2.0. Bioinformatics 2007, 23(21):2947-2948.

61. Huelsenbeck JP, Ronquist F: MRBAYES: Bayesian inference of phylogenetic trees. Bioinformatics 2001, 17(8):754-755.

doi:10.1186/1471-2164-15-667

Cite this article as: Bachmann et al.: Comparative genomics of koala, cattle and sheep strains of Chlamydia pecorum. BMC Genomics 2014 15:667.

\section{Submit your next manuscript to BioMed Central and take full advantage of:}

- Convenient online submission

- Thorough peer review

- No space constraints or color figure charges

- Immediate publication on acceptance

- Inclusion in PubMed, CAS, Scopus and Google Scholar

- Research which is freely available for redistribution 